Revista Brasileira de Agricultura Irrigada v.10, nº.5, p. 875 - 882, 2016

ISSN 1982-7679 (On-line)

Fortaleza, CE, INOVAGRI - http://www.inovagri.org.br

DOI: $10.7127 /$ rbai.v10n500457

Protocolo 457.16 - 01/09/2016 Aprovado em 14/10/2016

\title{
VARIABILIDADE ESPACIAL DA CAPACIDADE DE CAMPO E PONTO DE MURCHA PERMANENTE EM ARGILOSO AMARELO COESO
}

\author{
Joabe Martins de Souza ${ }^{1}$, Lucas Rosa Pereira ${ }^{2}$, Edvaldo Fialho dos Reis ${ }^{3}$, Robson Bonomo ${ }^{4}$
}

\begin{abstract}
RESUMO
O conhecimento da variabilidade espacial da capacidade de campo e ponto de murcha permanente é importante para a tomada de decisão no manejo da irrigação, visando suprir de forma correta a necessidade hídrica das culturas. Objetivou-se com esse trabalho avaliar a variabilidade espacial da capacidade de campo e ponto de murcha permanente em um Argissolo Amarelo Coeso cultivado com café Conilon no Norte Capixaba. Para avaliações de capacidade de campo (CC) e ponto de murcha permanente (PMP), foram retiradas amostras indeformadas, na linha e entrelinha da cultura, sendo quatros pontos, equidistantes em relação à cultura $(0,50$, 100 e $150 \mathrm{~cm}$ ), e cinco profundidades $0-20,20-40,40-60,60-80$ e 80-100 cm, com quatro repetições. Nas amostras foram tensões de 10 e $1.500 \mathrm{kPa}$, a CC e PMP, respectivamente. Os resultados obtidos foram submetidos à análise estatística descritiva e a dependência espacial foi avaliada pelos ajustes de variogramas. Para todos os eventos, os valores da média e mediana apresentaram-se, próximos entre si, mostrando distribuição simétrica, o que pode ser confirmado pelo coeficiente de assimetria nulo e de curtose próximos de zero. $\mathrm{O}$ índice de dependência espacial para CC e PMP foi forte para todos os modelos, porém o Gaussiano foi o melhor que se ajustou. A dependência espacial foi forte para CC e PMP, com alcance de 240 $\mathrm{cm}$ (CC) e $250 \mathrm{~cm}$ (PMP). A capacidade de campo e o ponto de murcha permanente apresentaram uma variabilidade espacial moderada podendo ser interpolados através dos métodos de krigagem com erros muito pequenos.
\end{abstract}

Palavras-chave: Geoestatística, Manejo da Irrigação, Dependência Espacial, Atributos hídricos do solo.

\footnotetext{
${ }^{1}$ Engenheiro Agrônomo, Mestre em Agricultura Tropical, Doutorando em Produção Vegetal, Universidade Federal do Espírito Santo, Centro de Ciências Agrárias e Engenharias, Programa de Pós Graduação em Produção Vegetal, Alto Universitário s/n, 29500-000, Alegre, ES. E-mail: joabenv@ gmail.com.

${ }^{2}$ Tecnólogo em Cafeicultura, Mestre em Produção Vegetal, Doutorando em Produção Vegetal, Universidade Federal do Espírito Santo, Centro de Ciências Agrárias e Engenharias, Programa de Pós Graduação em Produção Vegetal, Alto Universitário s/n, 29500-000, Alegre, ES. E-mail: lucasrosapereira@ hotmail.com

${ }^{3}$ Professor Associado do Departamento de Engenharia Rural, Centro de Ciências Agrárias e Engenharias, Universidade Federal do Espírito Santo, Alegre, ES, Brasil, edreis@cca.ufes.br

${ }^{4}$ Professor do Departamento de Ciências Agrárias e Biológicas, Centro Universitário Norte do Espírito Santo, Universidade Federal do Espírito Santo, São Mateus - ES, Brasil, robsonbonomo@gmail.com
} 


\title{
SPATIAL VARIABILITY FIELD CAPACITY AND PERMANENT WILTING POINT YELLOW CLAYEY COHESIONABSTRACT
}

\begin{abstract}
The knowledge of the spatial variability of field capacity and permanent wilting point is important for decision making in the management of irrigation, in order to meet properly the crop water requirement. The objective of this study was to evaluate the spatial variability of field capacity and permanent wilting point in a Cohesive Yellow Argisol cultivated with Conilon coffee in Norte Capixaba. For reviews of field capacity (CC) and permanent wilting point (PMP), undisturbed soil samples were taken on the line and leading culture, with four points, equidistant in relation to the culture $(0,50,100$ and $150 \mathrm{~cm})$ five depths $0-20,20-40$, 40-60, 60-80 and 80-100 cm, with four replications. All samples were tensions of 10 to 1500 $\mathrm{kPa}$, a CC and PMP, respectively. The results were submitted to descriptive statistical analysis and spatial dependence was assessed by variograms settings. For all events, the mean and median value is presented, close to each other, showing symmetrical distribution, which can be confirmed by the skewness coefficient zero and near zero kurtosis. The spatial dependence index for CC and PMP was strong for all models, but the Gaussian was the best set. The spatial dependence was strong for CC and PMP, with a range of $240 \mathrm{~cm}$ (CC) and $250 \mathrm{~cm}$ (PMP). The field capacity and the permanent wilting point showed a moderate spatial variability can be interpolated through kriging methods with very small errors.
\end{abstract}

Keywords: Geostatistics, Management of Irrigation, Space Dependence, hydric soil attributes.

\section{INTRODUÇÃO}

A agricultura irrigada tem sido uma importante estratégia para otimização da produção de alimentos, promovendo desenvolvimento sustentável no campo, com geração de emprego e renda (LUNA et al., 2013). O manejo da irrigação constitui uma técnica muito importante do ponto de vista econômico e ambiental numa atividade agrícola irrigada, proporcionando economia de água, energia, aumento da produtividade da cultura e melhoria na qualidade do produto (BONOMO et al., 2013).

Dentro dos vários atributos físico-hídricos dos solos relacionados ao armazenamento de água no solo e o desenvolvimento das plantas está à curva de retenção de água no solo (CRA). A curva de retenção de água no solo expressa a relação entre o potencial mátrico e a umidade do solo (NASCIMENTO et al., 2010),

A curva de retenção de água é usada em vários estudos como o balanço de água no solo, a disponibilidade de água às plantas, a dinâmica da água e solutos no solo, a infiltração e o manejo de irrigação. A CRA pode fornecer tanto o momento quanto a quantidade de água a aplicar para um manejo correto e adequado de irrigação. A partir dela, pode-se obter, também, os valores de umidade correspondentes à capacidade de campo $(\theta \mathrm{CC})$ e ao ponto de murcha permanente ( $\theta \mathrm{PMP})$, sendo que a diferença de umidade entre $\theta \mathrm{CC}$ e $\theta \mathrm{PMP}$ é definida como a capacidade de água disponível (CAD) de um solo a uma dada profundidade (BARRETO et al., 2011), sendo assim um auxilio ao produtor no manejo da irrigação.

O manejo da irrigação via solo segundo Dabach et al. (2016) é fundamentado no monitoramento do conteúdo e na disponibilidade de água no solo. Esse manejo tem como principio determinar a umidade do solo na zona do sistema radicular e repor o volume de água necessário até atingir a capacidade de campo do solo.

O conhecimento da variabilidade da umidade é de fundamental importância para que sistemas de irrigação por gotejamento sejam dimensionados (BATISTA et al., 2002), ainda segundo autores a variabilidade espacial da umidade necessita ser considerada, uma vez que o manejo da água está diretamente relacionado 
com as exigência hídricas das diversas culturas, com as características hidráulicas dos diferentes sistemas de irrigação utilizados e, principalmente, com a capacidade de retenção da água pelo solo, na profundidade efetiva do sistema radicular das culturas.

Diante disso o objetivou-se nesse trabalho avaliar a variabilidade espacial da capacidade de campo e ponto de murcha permanente em um Argissolo Amarelo Coeso cultivado com café Conilon no Norte Capixaba.

\section{MATERIAL E MÉTODOS}

O trabalho foi realizado em área cultivada com café Conilon (Coffea canephora Pierre) irrigado por gotejamento no município de São Mateus, localizado no Norte do Estado do Espírito Santo. O solo da área de estudo foi formado a partir de sedimentos argilosos da formação Barreiras na região dos Tabuleiros Costeiros, caracterizados por horizontes subsuperficiais adensados (IJSN, 2012), sendo classificado, após análise textural, como Argiloso Amarelo coeso, textura média em A e argilosa em B segundo metodologia da Embrapa (2013).

Para avaliações de capacidade de campo e ponto de murcha permanente, foram retiradas amostras indeformadas, com amostrador tipo Uhland, com 0,05 $\mathrm{m}$ de diâmetro e 0,03 cm de altura, na linha e entrelinha da cultura, sendo quatros pontos, equidistantes em relação à cultura $(0,50,100$ e $150 \mathrm{~cm})$, e em cada ponto a cinco profundidades 0-20, 20-40, 40-60, 60-80 e 80-100 cm, com quatro repetições.

Em seguida as amostras, foram levadas, sobre a membrana porosa, para o interior da câmara de pressão. Os pontos de tensão aplicados na câmara foram: 10 e $1.500 \mathrm{kPa}$, a referente a capacidade de campo e a ponto de murcha permanente, respectivamente, retirou-se a amostra da câmara após cessar a drenagem do excedente de umidade (equilíbrio entre a pressão aplicada e a umidade relacionada). Ao final das tensões aplicadas, as amostras foram levadas para estufa a $105{ }^{\circ} \mathrm{C}$ por 48 horas para a determinação da massa do solo seco, permitindo assim a obtenção das umidades em base gravimétricas correspondentes às tensões aplicadas.

Os resultados obtidos para a capacidade de campo e ponto de murcha permanente foram submetidos à análise estatística descritiva, calculando-se a média, mediana, valor mínimo, valor máximo, desvio padrão, coeficiente de variação, coeficiente de assimetria e de curtose, a fim de caracterizar a distribuição dos dados.

A dependência espacial foi avaliada pelos ajustes de variogramas, pressupondo a estacionariedade da hipótese intrínseca. Foram testados os modelos linear, gaussiano, esférico e exponencial. Foi ajustado o modelo que apresentou a menor soma dos quadrados do resíduo (SQR). Após a escolha do modelo, foram determinados os parâmetros: efeito pepita $\left(\mathrm{C}_{\mathrm{o}}\right)$, patamar $\left(\mathrm{C}_{\mathrm{o}}+\mathrm{C}\right)$ e alcance $(\mathrm{A})$. $\mathrm{O}$ índice de dependência espacial (IDE) foi calculado e classificado, segundo proposta de Zimback (2001), assumindo os seguintes intervalos: dependência espacial baixa para IDE $<25 \%$, moderada para $25 \%<$ IDE $<75 \%$ e forte para IDE $>75 \%$ (Equação 1).

$$
\mathrm{IDE}=\frac{\mathrm{C}}{\mathrm{C}+\mathrm{C}_{\mathrm{O}}} \times 100
$$

em que: $\mathrm{C}$ - semivariância estrutural ou espacial e $\mathrm{C}+\mathrm{C}_{\mathrm{o}}$ - patamar.

Uma vez detectada a dependência espacial, foi produzido o mapa de variabilidade do índice para os diferentes estádios estudados. A interpolação dos mapas foi realizada utilizando krigagem ordinária. Para as estimativas de valores em locais não amostrados, foram utilizados 16 vizinhos próximos e um raio de busca igual ao valor do alcance encontrado no ajuste do variograma. A análise geoestatística foi realizada utilizando o software GS+® "Geostatistical for Environmental Sciences", versão 7.0 (ROBERTSON, 2004), que utiliza os valores da variável de capacidade de campo e ponto de murcha permanente e suas respectivas coordenadas.

\section{RESULTADOS E DISCUSSÃO}


Os resultados referentes à análise estatística descritiva para os dados de capacidade de campo e ponto de murcha permanente são apresentados na Tabela 1, os quais indicaram distribuição normal. Para todos os eventos, os valores da média e mediana estão próximos entre si, mostrando distribuição simétrica, o que pode ser confirmado pelos valores do coeficiente de assimetria nulos e pelos de curtose próximos de zero se destacando para o ponto de murcha permanente, constando que a distribuição se aproxima da distribuição normal, a partir da qual toda inferência geoestatística pode ser realizada (CRESSIE, 1993).

Tabela 1. Análise descritiva da variável capacidade de campo (CC) e ponto de murcha permanente (PMP) para a malha com 144 em um Argiloso Amarelo Coeso.

\begin{tabular}{ccc}
\hline Análise Descritiva & \multicolumn{2}{c}{ Variável } \\
\cline { 2 - 3 } & CC & PMP \\
\hline Números de dados & 144 & 144 \\
Média & 0,263 & 0,193 \\
Mediana & 0,272 & 0,209 \\
Valor mínimo & 0,130 & 0,060 \\
Valor máximo & 0,350 & 0,280 \\
Desvio Padrão & 0,052 & 0,050 \\
Coeficiente de variação (\%) & 19,94 & 19,13 \\
Coeficiente de Assimetria & $-0,107$ & $-0,328$ \\
Coeficiente de curtose & 1,121 & 0,197 \\
\hline
\end{tabular}

Pode-se observar que, para a capacidade de campo e ponto de murcha permanente, a diferença entre a maior e a menor umidade é significativa, sendo de $0,220 \mathrm{~cm}^{3} \mathrm{~cm}^{-3}$ para ambas as variáveis estudadas e uma disponibilidade de água no solo igual a 0,700 $\mathrm{mm} \mathrm{cm}^{-3}$. Valores próximos ao encontrados de capacidade de campo e ponto de murcha permanente foram observados por Pacheco e Cantalice (2011) trabalhando com Argissolo Amarelo em Alagoas obtendo valores de médios de capacidade de campo e ponto de murcha permanente de $0,254 \mathrm{~cm}^{3} \mathrm{~cm}^{-3}$ e $0,189 \mathrm{~cm}^{3} \mathrm{~cm}^{-3}$ respectivamente. Já Guimarães et al. (2014) trabalhando com Argissolo Amarelo distróficos verificaram valores médios de $0,130 \mathrm{~cm}^{3} \mathrm{~cm}^{-3}$ para capacidade de campo e $0,064 \mathrm{~cm}^{3} \mathrm{~cm}^{-3}$ para o ponto de murcha permanente, resultado inferiores ao encontrados nesse trabalho.
Verificou-se, também, que o desvio quando comparados à média foi baixo para ambos as variáveis, tendo a baixa dispersão dos dados. Os coeficientes de variação mostraramse médios para as variáveis estudadas, classificado conforme Pimentel-Gomes (2009).

$\mathrm{Na}$ Tabela 2, onde estão presentes os valores dos parâmetros do semivariogramas de capacidade de campo e ponto de murcha permanente, verifica-se que o desempenho dos semivariogramas analisados pelos valores de coeficientes de determinação $\left(\mathrm{R}^{2}\right)$ variaram de 77 à $87 \%$ para capacidade de campo e 78 à 89 $\%$ para o ponto de murcha permanente, isso significa que mais de $77 \%$ (CC) e $78 \%$ (PMP) da variabilidade existente nos valores da semivariância estimada são explicadas pelos modelos ajustados.

Tabela 2. Parâmetros dos modelos de semivariogramas ajustados para capacidade de campo (CC) e ponto de murcha permanente (PMP).

\begin{tabular}{cccccccc}
\hline Variável & Modelo & Co & Co + C & Alcance & $\mathbf{R}^{\mathbf{2}}$ & SQR & IDE \\
\hline CC & Linear & 0,000096 & 0,005662 & 67,13 & 0,784 & $2,15 \times 10^{-6}$ & 98,3
\end{tabular}



ARGILOSO AMARELO COESO

\begin{tabular}{cccccccc} 
& Gaussiano & 0,001340 & 0,023770 & 244,21 & 0,870 & $1,29 \times 10^{-6}$ & 94,4 \\
& Exponencial & 0,000010 & 0,020020 & 622,50 & 0,759 & $2,40 \times 10^{-6}$ & 100 \\
& Esférico & 0,000060 & 0,012120 & 210,90 & 0,773 & $2,26 \times 10^{-6}$ & 99,5 \\
\hline \multirow{4}{*}{ PMP } & Linear & 0,000135 & 0,005443 & 67,13 & 0,808 & $1,69 \times 10^{-6}$ & 97,5 \\
& Gaussiano & 0,001320 & 0,023730 & 250,62 & 0,890 & $9,66 \times 10^{-7}$ & 94,4 \\
& Exponencial & 0,000010 & 0,019610 & 632,70 & 0,784 & $1,90 \times 10^{-6}$ & 99,9 \\
& Esférico & 0,000110 & 0,011590 & 210,90 & 0,797 & $1,78 \times 10^{-6}$ & 99,1 \\
\hline
\end{tabular}

Co - efeito pepita; Co + C - Patamar; SQR - Soma dos Quadrados do Resíduo; IDE - índice de dependência espacial $(<0,25$ Fraca; $>0,25<0,75$ (moderado); $>0,75$ forte).

O índice de dependência espacial para capacidade de campo e ponto de murcha permanente foi forte para todos os modelos, apresentando uma variação de 94 à $100 \%$ (CC) e 94 à $100 \%$ (PMP), porém o modelo que melhor se ajustou foi o Gaussiano que apresentou um maior coeficiente de determinação e menor soma dos quadros do resíduo para ambos as variáveis estudadas, resultado semelhante ao encontrado por Santos et al. (2012) trabalhando com umidade do solo em um Latossolo Vermelho. Já Nascimento et al. (2014) encontrou um melhor ajuste para a capacidade de campo e ponto de murcha permanente nos modelos exponencial na camada de 0-20 cm e Gaussiano na camada de $20-40 \mathrm{~cm}$.

Mion et al. (2012) avaliando a variabilidade espacial da umidade gravimétrica de um Argissolo Amarelo verificaram efeito pepita ou seja, não foi possível ajustar um modelo teórico aos semivariogramas experimentais, resultado que difere dos encontrados nesse trabalho. Já estudando a variabilidade espacial da umidade do solo em um Cambissolo Háplico, Campos et al. (2012; 2013) encontraram melhor modelo de ajuste o esférico, e Ávila et al. (2010) o modelo exponencial pelo método dos mínimos quadrados ponderados.

$\mathrm{O}$ alcance do modelo que melhor se ajustou foi $244 \mathrm{~cm}$ para capacidade de campo e $251 \mathrm{~cm}$ para ponto de murcha, neste caso o semivariograma mostra uma dependência espacial para a CC até $244 \mathrm{~cm}$ e PMP até 251 $\mathrm{cm}$, ou seja, amostras coletadas a distância inferiores possui dependência espacial e, no caso da utilização de métodos de análises estatísticas que consideram independência entre amostras, à distância de amostragem mínima deveria ser de o alcance obtido pelo modelo. Portando, segundo Batista et al. (2002) o alcance da dependência espacial da umidade e um parâmetro de fundamental importância para o planejamento e avalições de experimentos de irrigação, não somente para determinar a intensidade de amostragem, como também ajudar no planejamento de programas de irrigação, uma vez que detecta até onde uma variável em estudo apresenta dependência espacial.

Na Figura 1 temos o mapa da umidade de capacidade de campo e ponto de murcha permanente, onde se pode observar que a capacidade de campo até a profundidade de 15 $\mathrm{cm}$ está entre 0,170 à $0,193 \mathrm{~cm}^{3} \mathrm{~cm}^{-3}$, com alcance na entrelinha de até $87,5 \mathrm{~cm}$ da planta, sendo que a partir daí, tanto como em profundidade, a umidade do solo na capacidade de campo aumenta, o mesmo ocorrendo com o ponto de murcha permanente, mantendo-se a disponibilidade de água.

Com aumento da umidade do solo no ponto de murcha permanente, requer uma atenção por parte do produtor com o manejo da irrigação, já que, por exemplo, para café Conilon, o qual o solo em estudo e cultivado, Silva e Martins (2010) verificaram que as raízes de café Conilon se concentram na faixa mais superficial do solo, diminuindo em profundidade à medida que se aproximam da profundidade de 0,40 m. Partelli et al. (2014) também evidenciou os mesmo resultados, com uma concentração mais elevada na camada superior do solo $(0-10 \mathrm{~cm})$, o que diminui com a profundidade, apresentando concentrações mais baixas nas camadas abaixo de $40 \mathrm{~cm}$. 

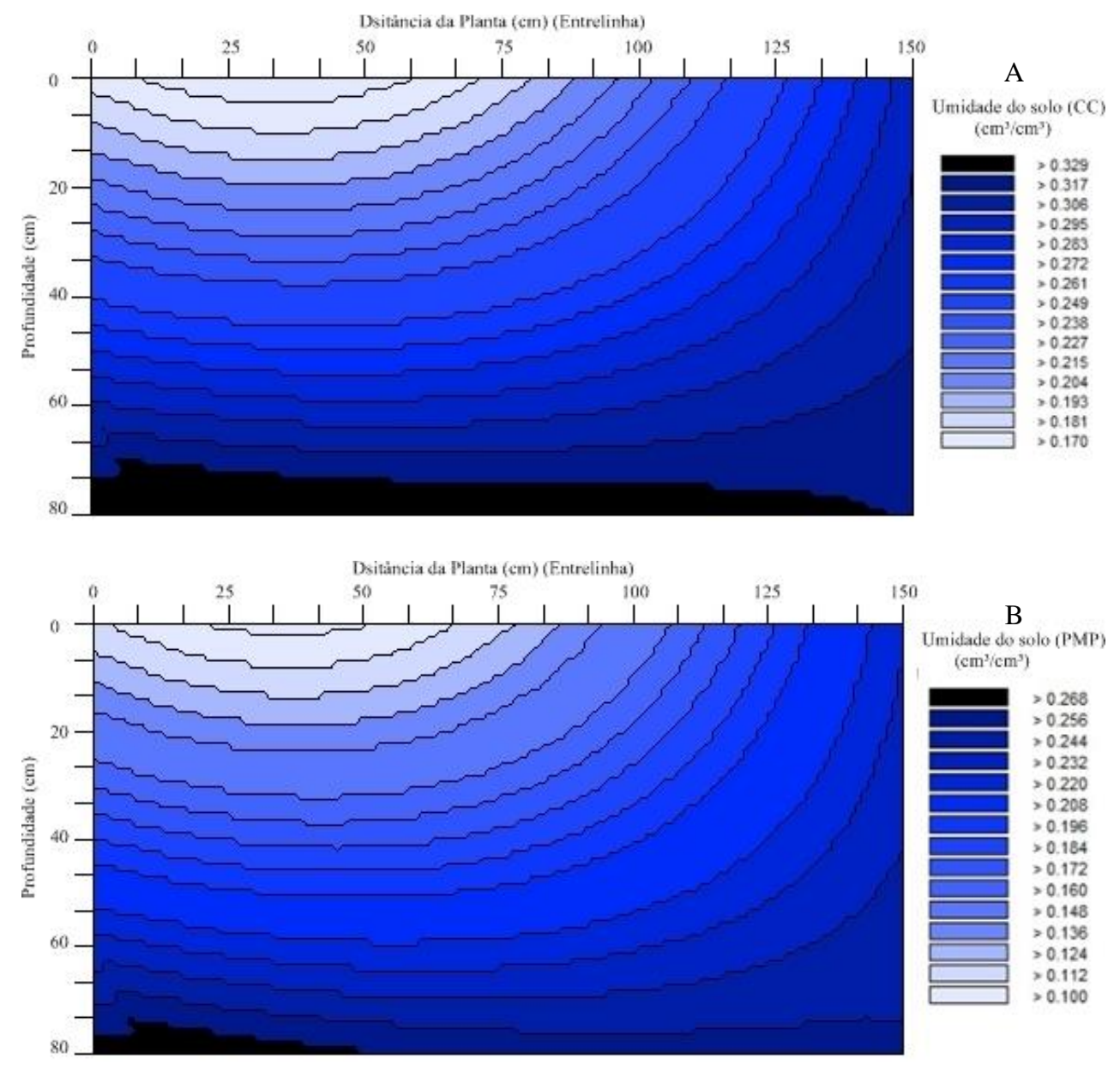

Figura 1. Mapas de krigagem da umidade na capacidade de campo (A) e ponto de murcha permanente (PMP) em um Argissolo Amarelo Coeso.

Esses resultados são importantes para o manejo da irrigação, principalmente para a irrigação por gotejamento, onde é possível verificar a variabilidade espacial da umidade do solo para esses dois parâmetros importantes para a irrigação, possibilitando planejar e manejar o sistema de irrigação corretamente, permitindo obtendo informações confiáveis para dimensionamento do bulbo molhado, e consequentemente das vazões e espaçamento dos gotejadores.

Para o cafeeiro Conilon, o planejamento e o manejo do sistema da irrigação são de suma importante em áreas antes não exploradas, se mostrando viável ao longo do tempo principalmente em regiões com distribuição de chuvas irregular, como no Norte do Estado do Espírito Santo. Com a água de irrigação se tornado um novo insumo da cafeicultura brasileira nos últimos anos, que tem a cada dia se tornado escasso, o seu uso racional e eficiente, através do uso de tecnologias associadas a irrigação como o uso do estudo da variabilidade dos atributo hídricos do solo e práticas adequadas de manejo, se torna indispensável na busca da minimização do uso de água, da preservação dos recursos hídricos, sem comprometer a produtividade e qualidade do cafeeiro.

\section{CONCLUSÕES}

A capacidade de campo e o ponto de murcha permanente apresentaram uma variabilidade espacial moderada com dependência forte, podendo ser interpolados através dos métodos de krigagem com erros muito pequenos.

A dependência espacial na entrelinha do cafeeiro foi de aproximadamente 240 e $250 \mathrm{~cm}$ para capacidade de campo e ponto de murcha 
permanente respectivamente, permitindo coletas de solo para determinação de umidade a distâncias maiores as citadas.

\section{REFERÊNCIAS BIBLIOGRÁFICAS}

ÁVILA, L. F.; MELLO, C. R.; SILVA, A. M. Continuidade e distribuição espacial da umidade do solo em bacia hidrográfica da Serra da Mantiqueira. Revista Brasileira de Engenharia Agrícola e Ambiental, Campina Grande, v.14, n.12, p.1257-1266, 2010.

BATISTA, I, F.; ZIMBACK, C. R. F.; VETTORATO, J. A. Variabilidade espacial da umidade do solo em irrigação por gotejamento sob cultivo protegido. Irriga, Botucatu, v.7, n.3, p.201-213, 2002.

BARRETO, H. B. F.; BATISTA, R. O.; FREIRE, F. G. C.; SANTOS, W. O.; COSTA, F. G. B. Análises de indicadores de retenção e armazenamento de água no solo do perímetro irrigado Gorutuba, em Janaúba-MG. Revista Verde, Mossoró, v.6, n.5, p.189-192, 2011.

BONOMO, D, Z.; BONOMO, R.; PARTELLI, F. L.; SOUZA, J. M.; MAGIERO, M. Desenvolvimento vegetativo do cafeeiro Conilon submetido a diferentes lâminas de irrigação. Revista Brasileira de Agricultura Irrigada, Fortaleza, v.7, n.2, p.157-169, 2013.

CAMPOS, M. C. C.; OLIVEIRA, I. A.; SANTOS, L. A. C.; AQUINO, R. E.; SOARES, M. D. R.; Variabilidade espacial da resistência do solo à penetração e umidade em áreas cultivadas com mandioca na região de Humaitá, AM. Revista Agro@mbiente Online, Roraima, v.6, n.1, p.9-16, 2012.

CAMPOS, M. C. C.; AQUINO, R. E.; OLIVEIRA, I. A.; BERGAMIM, A. C. Variabilidade espacial da resistência mecânica do solo à penetração e umidade do solo em área cultivada com cana-de-açúcar na região de Humaitá, Amazonas, Brasil. Revista Brasileira de Ciências Agrárias, Recife, v.8, n.2, p.305310, 2013.
CRESSIE, N. R. Statistics for spatial data. New York: A. Wiley- Interscience, 1993. 900p.

DABACH, S.; SHANIA, U.; LAZAROVITCH, $\mathrm{N}$. The influence of water uptake on matric head variability in a drip-irrigated root zone. Soil \& Tillage Research, Amesterdã, v.155, p.216224,2016.

EMPRAPA. Sistema brasileiro de classificação dos solos. 3.ed. Rio de Janeiro: Embrapa CNPS, 2013, 353p.

GUIMARÃES, D. V.; GONZAGA, M. I. S.; ARAÚJO, E. M.; NETO, J. O. M.; JÚNIOR, J. I. T. Impacto do cultivo de citros sobre a qualidade física de um Argissolo amarelo em Sergipe. Revista Caatinga, Mossoró, v. 27, n. 3, p. 183-189, 2014.

IJSN. Mapeamento geomorfológico do estado do Espírito Santo. Vitória, ES, 2012. 19f.: il. (Nota técnica, 28).

LUNA, N. R. S.; ANDRADE, E. M.; CRISÓSTOMO, L. A.; MEIRELES, A. C. M.; AQUINO, D. N. Dinâmica do nitrato e cloreto no solo e a qualidade das águas subterrâneas do distrito de irrigação Baixo Acaraú, CE. Revista Agro@mbiente Online, Roraima, v.7, n.1, p.53-62, 2013.

MION, R. L.; NASCIMENTO, E. M. S.; SALES, F. A. L.; SILVA, S. F.; DUARTE, J. M. L.; SOUZA, B. M. Variabilidade espacial da porosidade total, umidade e resistência do solo à penetração de um Argissolo amarelo. Semina: Ciências Agrárias, Londrina, v. 33, n. 6, p. 2057-2066, 2012.

NASCIMENTO, P. S.; SILVA, J. A.; COSTA, B. R. S.; BASSOI, L. H. Zonas homogêneas de atributos do solo para o manejo de irrigação em pomar de videira. Revista Brasileira de Ciência do Solo, Viçosa, v.38, n.4, p.11011113, 2014.

NASCIMENTO, P. S.; BASSOI, L. H.; PAZ SILVA, V. P.; PEDRO VAZ, C. M.; NAIME, J. M.; MANIERI, J. M. Estudo comparativo de 
métodos para a determinação da curva de retenção de água no solo. Irriga, Botucatu, v.15, n.2, p.193-207, 2010.

PACHECO, E. P.; CANTALICE, J. R. B. Compressibilidade, resistência a penetração e intervalo hídrico ótimo de um Argissolo Amarelo cultivado com cana-de-açúcar nos tabuleiros costeiros de alagoas. Revista Brasileira de Ciência do Solo, Viçosa, v.35, n.2, p.403-415, 2011.

PARTELLI, F. L.; COVRE, A. M.; OLIVEIRA, M. G.; ALEXANDRE, R. S.; VITÓRIA, E. L.; SILVA, M. B. Root system distribution and yield of 'Conilon' coffee propagated by seeds or cuttings. Pesquisa Agropecuária Brasileira, Brasília, v.49, n.5, p.349-355, 2014.

PIMENTEL GOMES, F. P. Curso de estatística experimental. 15. ed. Piracicaba: FEALQ, 2009. 451p.

ROBERTSON, G. P. GS+: Geoestatistics for the environmental sciences - GS+ User's Guide. Plainwell: Gamma Design Software, 2004. 152 p.

SANTOS, D.; SOUZA, E. G.; NÓBREGA, L. H. P.; BAZZI, C. L.; JÚNIOR, A. C. G. Variabilidade espacial de atributos físicos de um Latossolo Vermelho após cultivo de soja. Revista Brasileira de Engenharia Agrícola e Ambiental, Campina Grande v.16, n.8, p.843848, 2012.

SILVA, V. L. B.; MARTINS, P. F. S. Propriedades físicas do solo e sistema radicular do cafeeiro, variedade Conilon, sob diferentes espaçamentos. Revista Ciência Agrária, v.53, n.1, p.96-101, 2010.

ZIMBACK, C. R. L. Análise espacial de atributos químicos de solos para fins de mapeamento da fertilidade. $2001.114 \mathrm{f}$. Tese (Livre-Docência em Levantamento do solo e fotopedologia) - FCA-UNESP, Botucatu, 2001. 